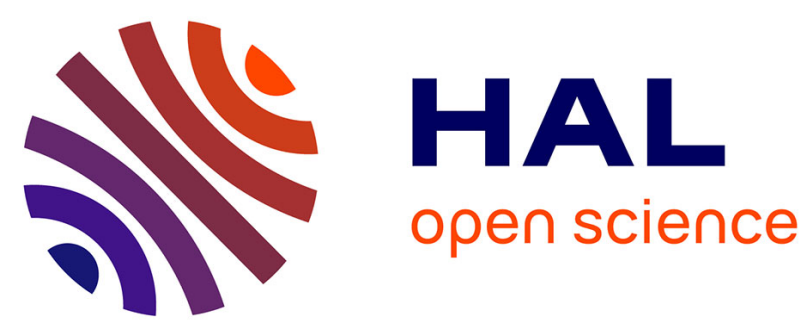

\title{
Correction of the Exon 2 Duplication in DMD Myoblasts by a Single CRISPR/Cas9 System
}

Annalisa Lattanzi, Stephanie Duguez, Arianna Moiani, Araksya Izmiryan, Elena Barbon, Samia Martin, Kamel Mamchaoui, Vincent Mouly, Francesco Bernardi, Fulvio Mavilio, et al.

\section{To cite this version:}

Annalisa Lattanzi, Stephanie Duguez, Arianna Moiani, Araksya Izmiryan, Elena Barbon, et al.. Correction of the Exon 2 Duplication in DMD Myoblasts by a Single CRISPR/Cas9 System. Molecular Therapy - Nucleic Acids, 2017, 7, pp.11-19. 10.1016/j.omtn.2017.02.004 . hal-02440767

\section{HAL Id: hal-02440767 https://hal.science/hal-02440767}

Submitted on 15 Jan 2020

HAL is a multi-disciplinary open access archive for the deposit and dissemination of scientific research documents, whether they are published or not. The documents may come from teaching and research institutions in France or abroad, or from public or private research centers.
L'archive ouverte pluridisciplinaire HAL, est destinée au dépôt et à la diffusion de documents scientifiques de niveau recherche, publiés ou non, émanant des établissements d'enseignement et de recherche français ou étrangers, des laboratoires publics ou privés. 


\title{
Correction of the Exon 2 Duplication in DMD Myoblasts by a Single CRISPR/Cas9 System
}

\author{
Annalisa Lattanzi, ${ }^{1}$ Stephanie Duguez, ${ }^{2,4}$ Arianna Moiani, ${ }^{1,5,6}$ Araksya Izmiryan, ${ }^{1,7,8}$ Elena Barbon, ${ }^{3}$ Samia Martin, ${ }^{1}$ \\ Kamel Mamchaoui, ${ }^{2}$ Vincent Mouly, ${ }^{2}$ Francesco Bernardi, ${ }^{3}$ Fulvio Mavilio, ${ }^{1}$ and Matteo Bovolenta ${ }^{1,3}$
}

${ }^{1}$ Genethon, INSERM UMR951, 1 bis, rue de l'Internationale BP60, 91002 Evry Cedex, France; ${ }^{2}$ Institut de Myologie, UMRS 974 Sorbonne Universités UPMC-INSERM, FRE 3617 CNRS, GH Pitié-Salpétrière 47 bd de l'Hôpital, 75651 Paris Cedex 13, France; ${ }^{3}$ Department of Life Sciences and Biotechnology, University of Ferrara, Fossato di Mortara 74, 44121 Ferrara, Italy

\begin{abstract}
Exonic duplications account for $10 \%-15 \%$ of all mutations in Duchenne muscular dystrophy (DMD), a severe hereditary neuromuscular disorder. We report a CRISPR (clustered regularly interspaced short palindromic repeat)/Cas9-based strategy to correct the most frequent (exon 2) duplication in the DMD gene by targeted deletion, and tested the efficacy of such an approach in patient-derived myogenic cells. We demonstrate restoration of wild-type dystrophin expression at transcriptional and protein level in myotubes derived from genome-edited myoblasts in the absence of selection. Removal of the duplicated exon was achieved by the use of only one guide RNA (gRNA) directed against an intronic duplicated region, thereby increasing editing efficiency and reducing the risk of off-target effects. This study opens a novel therapeutic perspective for patients carrying disease-causing duplications.
\end{abstract}

\section{INTRODUCTION}

Duchenne muscular dystrophy (DMD; OMIM \#310200) is an $\mathrm{X}$-linked recessive muscle-wasting disease, characterized by progressive weakening of skeletal, respiratory, and cardiac muscle followed by necrosis and fibrosis. DMD affects $~ 1: 4,087$ live male births and is associated with delayed motor milestones. ${ }^{1} \mathrm{DMD}$ is caused by absent or reduced levels of the dystrophin protein, which plays an important role in the stabilization of sarcolemma. In the absence of dystrophin, muscle strength steadily declines in the first decade of life, accompanied by progressive degeneration and regeneration of muscle fibers and their replacement by fat and connective tissue. ${ }^{2}$ Lack of dystrophin affects as well the cardiac muscle and the diaphragm, resulting in respiratory and heart failure and premature death.

DMD occurs as a result of mutations in the DMD gene that lead to premature termination of translation. The most frequent mutations are exonic deletions and duplications that induce a frameshift in the protein-coding sequence. ${ }^{3}$ Skipping of in-frame mutated exons and deletions by antisense oligonucleotides (AONs) has long been considered a promising therapeutic strategy for DMD. This strategy aims at artificially inducing favorable exclusion of mutated/out-of-frame exons to restore the correct reading frame. ${ }^{4}$
The exciting prospect of an effective AON-based exon skipping therapy was raised by promising data from early clinical trials that were, however, not confirmed by double-blind, placebocontrolled phase IIb and III trials, which failed to meet efficacy endpoints. ${ }^{5}$ Independently from its efficacy, AON-based exon skipping can target only a limited number of mutations: skipping of exon 51, which could restore a correct reading frame in the largest proportion of DMD mutations, would be applicable to just $\sim 10 \%$ of DMD patients. ${ }^{6,7}$ Moreover, this and similar treatments are not applicable to out-of-frame mutations and are of limited efficacy for exon duplications, ${ }^{8,9}$ therefore requiring a multi-exon skipping approach. $^{10}$

Among the reported DMD mutations, duplications represent a distinct group accounting for $\sim 10 \%-15 \%$ of those reported in the Leiden database, ${ }^{2}$ although their incidence may be higher, ${ }^{11}$ Duplications have been generally neglected by therapeutic approaches. Wein et al. ${ }^{12}$ recently reported a successful attempt at inducing out-offrame skipping of exon 2, causing an alternative translation initiation in exon 6 and leading to expression of a functional N-truncated dystrophin. These results support a potential therapeutic approach for patients with mutations within the $5^{\prime}$ exons of DMD, but it would be ineffective for all the other duplications.

There is no definitive cure for $\mathrm{DMD}$, and for a long time the only treatment option has been corticosteroid administration, a palliative management associated with many side effects. However, two drugs have recently obtained conditional approval in Europe

Received 12 November 2016; accepted 6 February 2017; http://dx.doi.org/10.1016/j.omtn.2017.02.004.

${ }^{4}$ Present address: Northern Ireland Centre for Stratified Medicine, Biomedical Sciences Research Institute, University of Ulster, C-TRIC Building, Altnagelvin Area Hospital, Glenshane Road, BT47 6SB Derry/Londonderry, Ireland

${ }^{5}$ Present address: Institut Curie-26, Rue d'Ulm, 75248 Paris, France

${ }^{6}$ Present address: CNRS UMR 3215, INSERM U934, 75248 Paris, France

${ }^{7}$ Present address: INSERM UMR 1163, Imagine Institute, 75015 Paris, France

${ }^{8}$ Present address: University Paris Descartes Sorbonne Cité, 75015 Paris, France

Correspondence: Matteo Bovolenta, Department of Life Sciences and Biotechnology, University of Ferrara, Via Fossato di Mortara 74, 44121 Ferrara, Italy. E-mail: bvlmtt@unife.it 


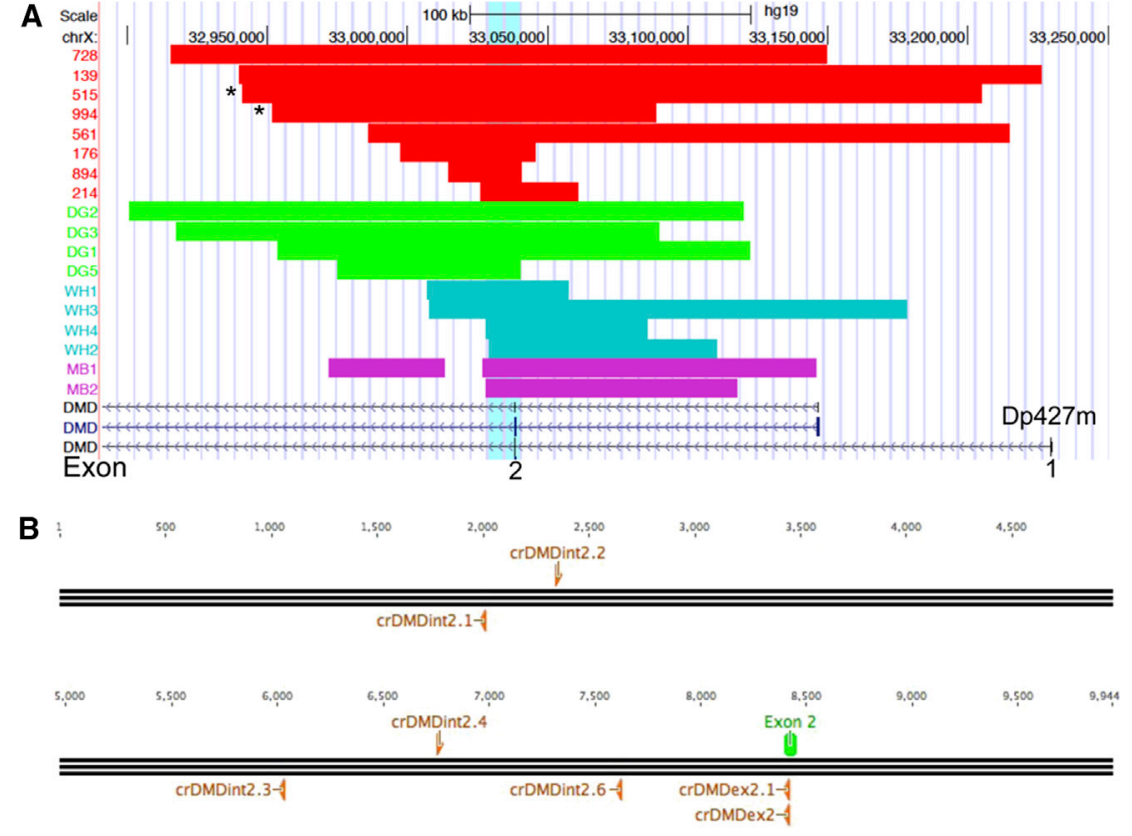

Figure 1. CGH Profile of DMD Exon 2 Duplications and CRISPR Design

(A) Breakpoint analysis of DMD exon 2 duplications from different cohorts (Cochin, red; Bovolenta et al., ${ }^{29,30}$ purple, DelGaudio et al.,, ${ }^{31}$ green; White et al., ${ }^{32}$ turquoise) allowed us to identify the minimal common duplicated region (light blue shade); (B) Diagram representing the $\sim 10 \mathrm{~kb}$ of the minimal common duplicated region and the position and orientation of the gRNAs (orange) designed with ZiFiT website in respect to the exon 2 of the DMD gene (green). The asterisks $\left(^{*}\right)$ indicate the mutation of the two patients tested in the present study.

on the efficiency of the gene-editing approach and the correct localization of the resulting protein.

\section{RESULTS}

\section{Strategy for the Correction of Duplications in DMD and gRNA Rational Design}

Our strategy to target duplications relies on the hypothesis that a single guide RNA (gRNA)

(Ataluren, a read-through drug for premature stop mutations) ${ }^{13}$ and the United States (Eteplirsen, an antisense molecule to restore the open reading frame in patients with mutations correctable by skipping exon 51). ${ }^{14}$

Genome editing technology uses synthetic nucleases to introduce targeted modification at specific loci in the genome by exploiting the endogenous cellular DNA repair mechanisms. These engineered enzymes are commonly based on zinc finger nucleases (ZFNs), ${ }^{15}$ transcription activator-like effector nucleases (TALENs), ${ }^{16}$ meganucleases, ${ }^{17}$ and, lately, the RNA-guided clustered regularly interspaced short palindromic repeat (CRISPR)/Cas9 system. ${ }^{18}$ These tools have been used to create new animal models ${ }^{19-21}$ and, recently, in vitro and in vivo therapeutic strategies for $\mathrm{DMD}^{22-25}$ aimed at restoring disrupted reading frames by deleting instead of skipping mutated/ out-of-frame exons. Compared to AON-mediated exon skipping, which involves repeated injection of the therapeutic molecules with toxicity and variable tissue uptake, ${ }^{26,27}$ the exon deletion strategy would require only one administration of the gene-editing system to achieve full exclusion of the targeted exon. Restoration of the reading frame for some duplication by excising one copy of the duplicated exon could permit expression of a normal dystrophin transcript, as recently demonstrated for a multi-exonic duplication of $139 \mathrm{~kb}^{28}$ and allow synthesis of a normal dystrophin isoform as compared to the restoration of the reading frame around the more common deletions that can generate a less severe, Becker muscular dystrophy (BMD)-like phenotype.

We report a CRISPR/Cas9 strategy to remove the most frequent duplication in the DMD gene, applicable to all patients with exon 2 duplications. The deletion restores synthesis of wild-type dystrophin at the transcriptional and protein level, adding relevant information designed over a tandem duplicated region will cut twice, leading to the deletion of the region between the two double-strand breaks (DSBs), thereby removing the duplication. This approach has been independently validated by the Cohn lab (University of Toronto) to remove a duplication of the exons $18-30$ in the DMD gene. ${ }^{28}$

To identify editing targets able to correct the DMD duplication of the exon 2 (dup2) in all patients bearing this type of mutation, we collected DNA from eight patients and characterized the extension of the copy number variation with the DMD-CGH array. ${ }^{29}$ Data from ten more patients were collected from the literature ${ }^{29-32}$ and aligned with the other duplications analyzed in order to identify a region shared among all the patients studied (Figure 1A). Interestingly, we identified duplicated regions spanning 25 to $286 \mathrm{~kb}$ with no common breakpoints.

A duplicated minimum common region (MCR) of about $10 \mathrm{~kb}$ was uploaded in ZiFiT and gRNAs of 18 nucleotides were selected based on the available guidelines and on the smallest number of potential off targets with a maximum of two mismatches. We also designed two gRNAs (one of which was $19 \mathrm{nt}$ ) targeting the coding sequence of the exon 2 in order to confirm the evidence that shifting the reading frame in the early codons should trigger the translational restart at exon 6 as previously demonstrated by an exon skipping approach (Figure 1B). ${ }^{12}$

gRNAs were named according to the following code: cr (crispr), $\mathrm{DMD}$, int (intron), or ex (exon) 2 and numbered from 1 to 6 (the crDMD notation have been omitted in Figures $2 \mathrm{E}, 2 \mathrm{~F}$, and 3 to limit redundancy). Repeated regions and functional splicing sequences 

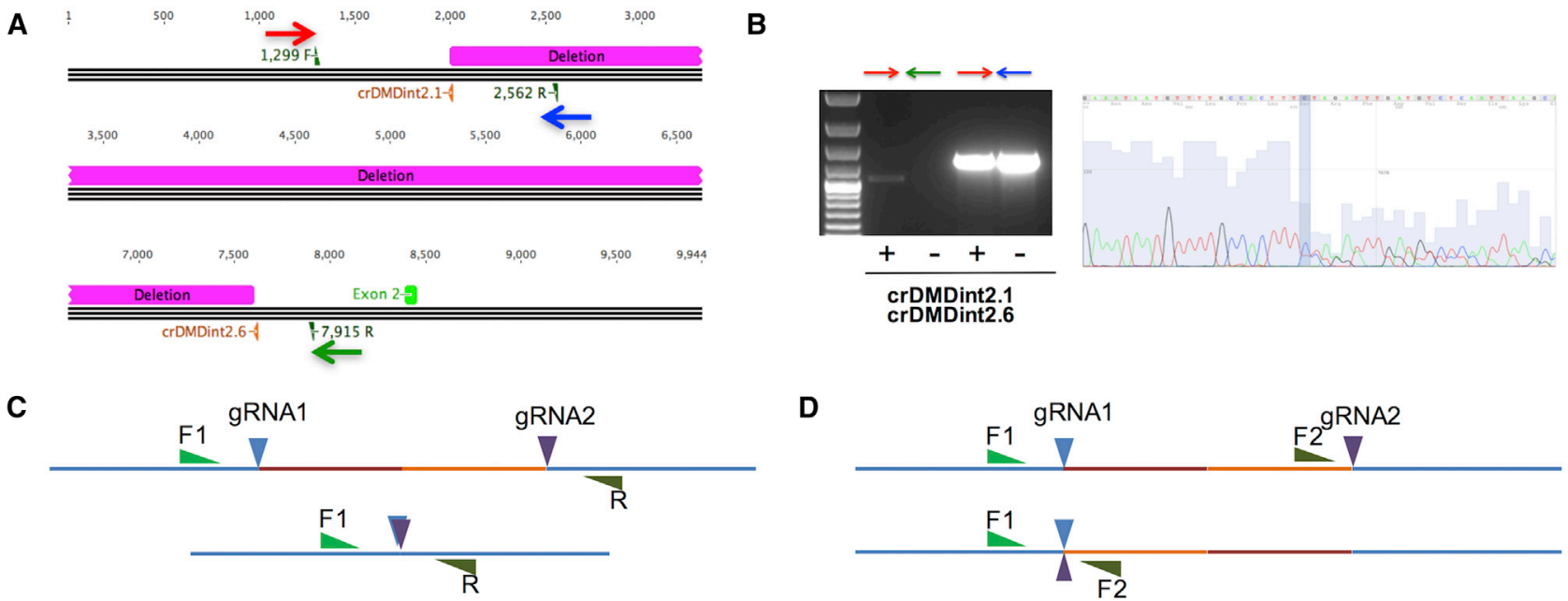

D
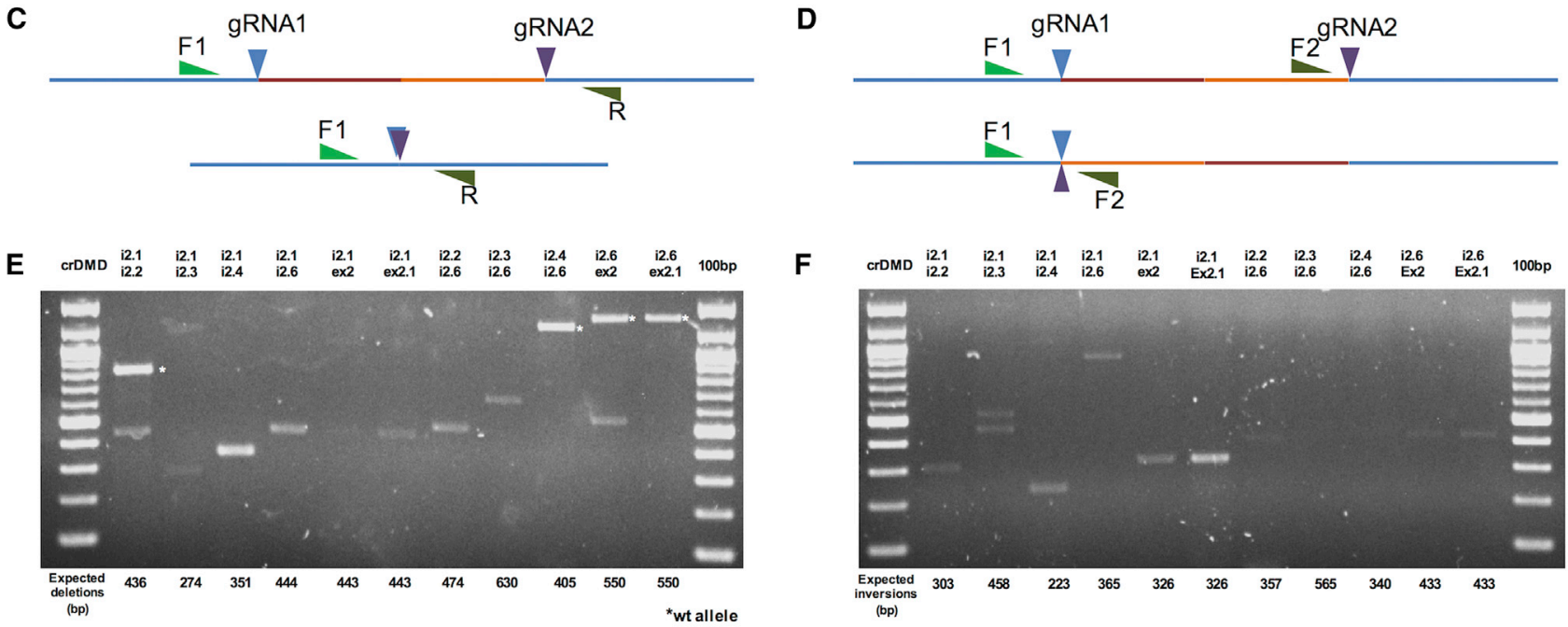

Figure 2. Detection of Deletion and Inversions after Transfection of Two gRNAs in Myogenic Cells

(A) Schematic representation of the 5.6-kb deletion created by transfecting the crDMDint2.1 and int2.6 gRNAs in a control myogenic cell line. The primers used to amplify across the deletion breakpoint and the wild-type sequence are shown as colored arrows: red, forward common primer; blue, wild-type-specific reverse primer; green, deletion-specific reverse primer. (B) PCR amplification and sequencing of the wild-type and deleted products. The PCR amplification revealed a product corresponding to the deleted sequence of about $1.1 \mathrm{~kb}$ only in treated cells (left panel, first lane), whereas the amplification of the wild-type sequence was obtained in both treated and untreated cells with a reduced amplification evident in treated cells. Sequence analysis of the deleted product showed the presence of two products with the only difference of a thymine at the breakpoint causing a shifting starting from the shaded column (right panel). (C and D) Schemes representing the strategies used to amplify the deletions and inversions caused by two gRNAs. The same forward primer located upstream the first gRNA binding site (F1) was used in both approaches in combination with a reverse one downstream to the second gRNA binding site for deletions detection (C) and a second forward primer (F2) upstream the second gRNA binding site for inversions amplification (D). (E) PCR products resulting from the amplification of the deletion breakpoints. The combination of the gRNAs used is reported above the image, whereas the expected products size in base pairs is displayed below it. All the bands where excised and sequenced or directly sequenced (Figure S2). (F) PCR products resulting from the amplification of the inversions breakpoints. The combination of the gRNAs used is reported above the image, whereas the expected products size in base pairs is displayed below it. All the bands where excised and sequenced or directly sequenced (Figure S2). A PCR product resulting from the nonspecific amplification was found in the second lane (i2.1/i2.3, upper band). An unexpected product was found in the fourth lane upon treatment with i2.1 and i2.6; sequencing reported the presence of a complex rearrangement and coexistence of an inversion and deletion between the two gRNAs (Figure S2). T, treated; UT, untreated.

were excluded from the design. All gRNA sequences were cloned in the MLM3636 plasmid backbone (Table S1).

\section{Testing of gRNAs Activity by Plasmid Transfection in HEK293T and Myogenic Cell Lines}

To validate the ability of gRNAs to induce DSBs, we transfected each gRNA and the Staphylococcus pyogenes's Cas9 (spCas9, JDS246) in HEK293T cells and performed the T7E1 assay. Interestingly, the PCR system amplifying across the gRNA crDMDint2.1 and crDMDint2.2 regions incorporates a simple tandem repeat (TG) that creates a hetero- duplex that is cleaved into two fragments when digested with the T7E1 enzyme (Figure S1).

To test the gRNAs in myogenic cells, we obtained primary myoblasts from two DMD patients with large duplications of 137 and $263 \mathrm{~Kb}$, respectively, encompassing the exon 2 (Figure 1A; \#994 and \#515). These cells have been immortalized as previously described. ${ }^{33}$ Due to low transfection efficiency ( $<20 \%$, data not shown) of myoblasts, we chose to test the presence of deletions caused by two gRNAs, as they can be easily discriminated from the wild-type sequences. Hence, 
A

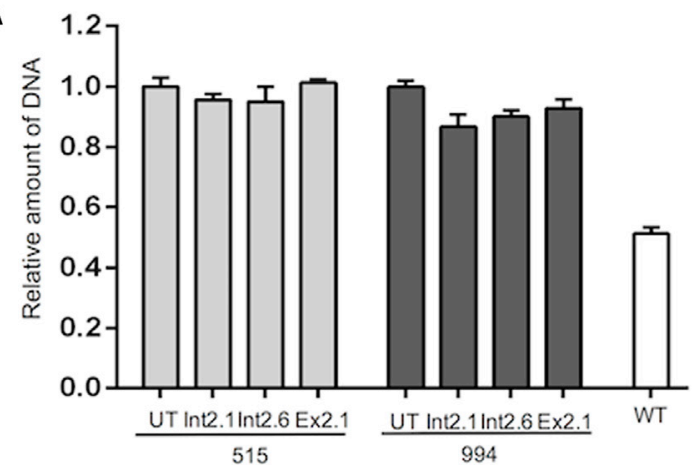
$\mathbf{C}_{427 \mathrm{kD}}$ $100 \mathrm{kD}$
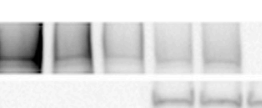

B

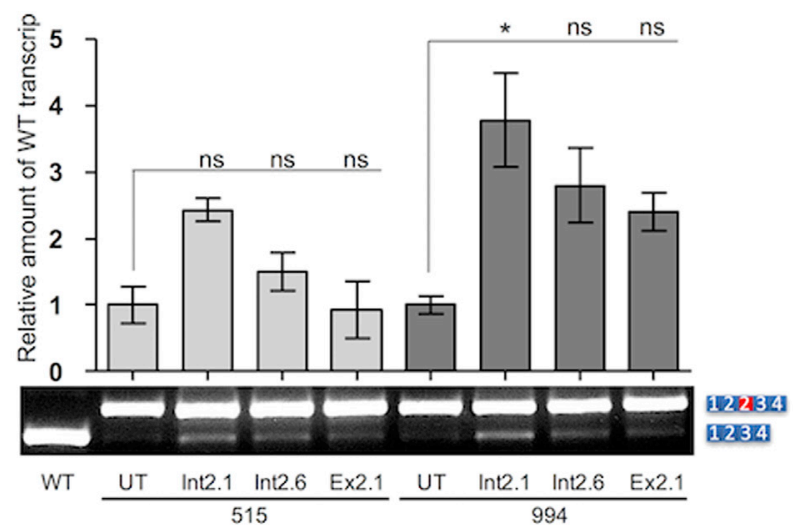

Dystrophin D

Cas9

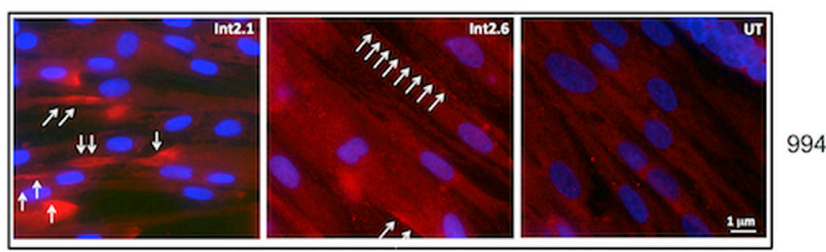

Figure 3. DNA, RNA, and Protein Expression Analysis after Infection with LentiCRISPRv2

(A) Histograms reporting the genomic analysis by real-time PCR of the duplicated sequence. Transduced cells present reduction of the duplication in respect to untreated cells (UT) set to 1. Wild-type cells were analyzed as positive control. (B) RT-PCR was performed with primers designed on the exon 1 and 4 . One microliter of the displayed PCR was loaded on a high-sensitivity DNA chip and run on the Bioanalyser 2100 (Agilent). Quantification of the peak obtained was performed calculating the ratio of the area of the normal transcript and the sum of the area of normal and duplicated transcript multiplied for 100 and normalized by setting the untreated (UT) as one. ${ }^{*} p<0.05$ (Kruskal-Wallis test with post hoc Mann-Whitney test from three independent experiments). ns, not significant. (C) Western blot from treated and untreated myotubes of patient 994 and 515 , with antibodies against dystrophin, Cas 9 and $\alpha$-actinin as loading normalizer. The amount of dystrophin was normalized to that of actinin by densitometric analysis. WT, wild-type. (D) Immunocytochemistry of dystrophin (red) and nuclei (blue) in myotubes from patient 994 . Scale bar, $1 \mu \mathrm{m}$. All error bars represent the SD for three independent experiments.

we co-transfected Cas9 and different combinations of two gRNA in a wild-type myogenic cell line and tested the ability of the two gRNAs to delete a genomic region. We first tested the two gRNA crDMDint2.1 and crDMDint 2.6 and detected a deletion of $5.6 \mathrm{~kb}$ resulting in about a $20 \%$ of reduction in the amplification of the wild-type region in treated cells when compared to untreated cells (Figure 2A). The PCR product corresponding to the deleted sequence was only detectable in treated cells. Sequencing of the latter revealed the coexistence of two products differing from each other for the insertion of one thymine at the breakpoint (Figure 2B).

As previously reported by Kraft et al., ${ }^{34}$ the use of two synthetic gRNAs targeting two different positions of the same chromosome induces deletions, inversions and duplications through non-homologous end joining (NHEJ). Hence, we tested the occurrence of deletions and inversions by specifically designed PCR (Figures 2C and 2D) amplifying and sequencing the expected deletions and inversions of different range between the target sites of the tested gRNAs (Figures $2 \mathrm{E}$ and $2 \mathrm{~F}$ ). A representation of deletions and inversions obtained with two gRNAs and the sequence of the respective breakpoints is available in Figure S2.

Overall, five out of seven of our gRNAs resulted to have activity in HEK293T cells (Figure S1) and were able to introduce deletions $(80 \%)$ and inversions $(72 \%)$ when used in couples in a myogenic cell line (Figure 2).

\section{Targeted Removal of a Large Duplication in Myogenic Cells by Lentiviral Delivery of CRISPR/Cas9 without Selection}

In order to increase the efficacy and maximize our results in cell lines, we cloned the more efficient gRNAs in the LentiCRISPRv2 vector (Addgene Plasmid \#52961). ${ }^{35}$ We did not select the infected cells by antibiotics resistance as the immortalization process takes advantage of two lentiviruses and one shares the puromycin resistance also present in the LentiCRISPRv2 vector. We analyzed vector copy number (VCN) $72 \mathrm{hr}$ after transduction at a multiplicity of infection (MOI) of 20 (Table 1). \#515 and \#994 cells received an average of 0.6 and 2.7 copies of LentiCRISPRv2, respectively. 


\begin{tabular}{|c|c|c|c|}
\hline \multicolumn{4}{|c|}{ Table 1. VCN Analysis } \\
\hline Cells & LV.CRISPR & VCN (Total) & VCN (LV.CRISPR) \\
\hline 515 & - & 3.4 & - \\
\hline 994 & - & 3.6 & - \\
\hline 515 & LV_int2.1 & 4.1 & 0.7 \\
\hline 515 & LV_int2.6 & 3.8 & 0.4 \\
\hline 515 & LV_ex2.1 & 4.2 & 0.8 \\
\hline 994 & LV_int2.1 & 6.6 & 3 \\
\hline 994 & LV_int2.6 & 6.2 & 2.6 \\
\hline 994 & LV_ex2.1 & 6.2 & 2.6 \\
\hline
\end{tabular}

To quantify the deletions of the duplicated region and indirectly the wild-type alleles created by the editing, we analyzed the genomic region targeted by our approach versus a region in intron 67 of the DMD gene by real-time PCR. We detected the highest rate of deletions in 994 cells treated with LV_int2.1 (about the 13\%), whereas no deletions at all were revealed in 515 cells transduced with LV_Ex2.1 (Figure 3A).

We then investigated the presence of inversion events by amplifying the region targeted by the gRNAs with only one primer close to the target region (Figure S3). We obtained no evidence of inversion event despite 45 cycles of PCR amplification (data not shown).

In order to better investigate dystrophin expression, we induced myoblast differentiation to myotubes. RNA analysis revealed an increased ratio between wild-type/duplicated transcripts ranging from 1.5 to about 4 -fold in cells transduced by LentiCRISPRv2 (Figure $3 \mathrm{~B}$ ). Western blot analysis showed restoration of wild-type dystrophin in \#994 cells transduced with LV_int2.1 (11\%) and LV_int2.6 (7\%) when compared to wild-type (Figure 3C), whereas \#515 cells did not show any labeling; this is probably due to the low rate of transduction in \#515 cells with VCN spanning from 0.4 to 0.8 while being around 3 in \#994 cells.

Immunocytochemistry showed intense expression and correct localization of dystrophin in clustered myotubes in differentiated \#994 cells infected with LV_int2.1 and LV_int2.6, even though these represented only a $5 \%$ of total cell population (Figure $3 \mathrm{D}$ ).

We then investigated the on-target versus off-target activity of gRNA crDMDint2.1, which showed the highest activity in restoring dystrophin expression. We scored $\sim 73 \%$ on-target activity in 110 clones sequenced (Figure S4, upper table) and no off-target effect by T7E1 assay (Figure S4, bottom table).

\section{DISCUSSION}

The recent development of the CRISPR/Cas9 system have brought great excitement in the research community and new perspectives for the treatment of several genetic diseases, and recently for DMD with convincing data in vitro and in vivo. ${ }^{36-40}$ For these purposes, the CRISPR/Cas9 system has been mainly exploited to remove outof-frame or in-frame mutated exons and restore the dystrophin reading-frame by targeting the sequence to be removed with two gRNAs. This approach is applicable to out-of-frame deletions and point mutations optimistically accounting for the $85 \%$ of all mutations, restoring the expression of a smaller functional protein and converting the severe phenotype into a milder, Becker-like one, as aimed by exon skipping approaches. ${ }^{41}$

Duplications represent the third cause of DMD among all the dystrophin mutations. The attempt to treat duplications has been recently reported, in a multi-exonic duplication in the DMD gene. ${ }^{28}$ Here, we described a strategy to correct the most frequent duplication in the DMD gene that could be extended to all other, less frequent duplications, leading to the synthesis of full-length, wild-type dystrophin. CGH analysis allowed us to identify a minimal region that is duplicated and shared among all the patients studied, against which we screened gRNAs with the aim of finding a deletion strategy potentially addressing all those patients (Figure 1). We used lentiviral vectors to bring our gRNAs and the spCas9 in patient-derived myoblasts, in order to maximize the efficiency of delivery and have both elements in a single vector. We were able to quantify the efficiency of the Cas9-induced deletions directly in treated cells without selection, and assay the results at both transcriptional and protein expression levels by cytochemical analysis of myotubes differentiated in vitro from treated myoblasts. The sequence analysis of inversions and deletions breakpoints indicated that rearrangements caused by two gRNAs are mostly neat and show the mutational spectrum introduced by NHEJ only in few cases (Figure S2). Interestingly, we did not detect inversions when dup2 patient's myoblasts were treated, backing up the concept that deletions occur more frequently than inversions in our system, as described elsewhere. ${ }^{34}$

Treatment of immortalized myoblasts derived from two dup2 patients resulted in the deletion of the duplication at the genomic level (Figure 3A) and in an increased expression of wild-type dystrophin transcripts (Figure 3B). This expression was followed by a detectable and correctly localized dystrophin only in the \#994 cell line (Figures 3C and 3D), which carries a smaller duplication of $137 \mathrm{~kb}$, and not in the \#515 cell line, which carries a larger, 263$\mathrm{kb}$ duplication, probably due to the limited transduction efficiency in this cell line. Our results are concordant with those reported by Wojtal et al. $^{28}$ in terms of restoration of protein expression in cells bearing deletions of comparable size (139 and $137 \mathrm{~kb}$ ). We extended the significance of those findings by reporting the correct localization of dystrophin at the sarcolemma in myotubes derived from \#994 cells transduced with two out of three lentiviral vectors, despite the small number of myoblasts positive for DMD expression (Figure 3D).

Even if we did not detect off-target activity for the best gRNA in our hands (Figure S4, bottom table), the recent improvements in the CRISPR design, ${ }^{42}$ specificity of the spCas $9^{43}$ and repertoire of Cas 9 
enzymes ${ }^{44,45}$ let foresee a safer application of the technology, which opens perspectives for the clinical investigation.

In fact, by delivering Cas9 and gRNAs with adeno-associated virus (AAV) vectors it will be possible to specifically drive the correction of duplications to the muscles in vivo. ${ }^{37}$ It has been demonstrated that the AAV viral genomes are lost in the regenerating dystrophic skeletal muscle. ${ }^{46}$ This could mimic a transient expression of the CRISPR/Cas9 system, which reduces the risk of immune response and off-target activity while still producing stable genome modifications in satellite cells that could be able to repair or restore the muscles. ${ }^{37}$

Taken together, these data indicate that the removal of a duplication event by Cas9-mediated genome editing is feasible with only one gRNA directed against a duplicated intronic region (which is duplicated in a relevant number of DMD patients), increasing editing efficiency and reducing the risk of off-target effects. These findings provide proof of concept for a gene-editing approach in DMD patients carrying duplications and lay out the foundation for further research into the application such an approach in other inherited disorders caused by gene duplications.

\section{MATERIALS AND METHODS \\ Patients}

We have studied eight DMD patients known to have duplications of the exon 2 by multiple ligase probe amplification (MLPA) analysis. DNA from these patients was obtained thanks to the collaboration with Dr. France Leturcq through the AFM-Telethon Biobank.

Myoblasts from two of these patients (ID \#515 and \#994), available through the Biobank, were immortalized thanks to the collaboration with the Institut de Myologie as previously described. ${ }^{33}$

Informed consent was obtained from all patients to conduct the study, which was in accordance with the Helsinki Declaration. All the experimental procedures were approved by Genethon.

\section{CGH Analysis}

According to manufacturer's instructions, DNA samples were processed and hybridized to the DMD-CGH array as previously reported. ${ }^{29}$ The array was analyzed with the Agilent scanner and the Feature Extraction software (v.12.0). A graphical overview and analysis of the data were obtained using the Agilent Genomic Workbench (v.7.0). For identifying duplications and deletions, we used the standard setup of the ADM-2 statistical analysis provided by Genomic Workbench software (Agilent).

The array platform and data-related information have been submitted to the online data repository Gene Expression Omnibus (GEO, NCBI): GSE83658.

A graphical representation of the duplications was produced by creating a custom track and displaying it in UCSC Genome Browser GRCh37/ hg19 assembly (http://genome.ucsc.edu). Ten more characterized duplications of the exon 2 from DMD patients were collected from the literature ${ }^{27-30}$ and represented with a custom track (Figure 1A).

\section{gRNA Design and Off-Target Prediction}

The minimal common duplicated region shared among all the patients was uploaded in ZiFiT (http://zifit.partners.org/ZiFiT/) and used as a template to design six truncated gRNAs ${ }^{47}$ of $18 \mathrm{bp}$ and one (crDMDex2) of $19 \mathrm{bp}$ (Figure 1B). Oligos used to clone the gRNAs are shown in the Table S1. According to the available guidelines ${ }^{48}$ on CRISPR design we selected and cloned into the MLM3636 backbone (Addgene number 43860) those with less than ten off-target regions with two mismatches as predicted by ZiFiT (Figure S4).

\section{Cell Cultures}

To preliminarily screen the activity of the candidate gRNAs, we transfected HEK293T cells with the Cas9 plasmid JDS246 (Addgene number 43861) and with each gRNA, using Lipofectamine2000 (Thermo Fisher Scientific). DNA extraction (Blood \& Cell Culture DNA Mini Kit, QIAGEN) was performed $72 \mathrm{hr}$ after transfection for PCR amplification and T7E1 assay.

Immortalized DMD myoblasts were maintained in Skeletal Muscle Growth Medium (Promocell) and differentiated into myotubes for at least 7 days in Skeletal Muscle Differentiation Medium (Promocell) at $37^{\circ} \mathrm{C}$ with $5 \% \mathrm{CO}_{2}$ incubation. Transfection of spCas 9 and DMD gRNAs was performed with JetPEI (Polyplus) following manufacturer's instructions. After $72 \mathrm{hr}$, DNA was extracted to perform PCR amplification.

\section{T7EI Assay}

PCR amplicons for targeted and off-target genomic regions were obtained by amplification with Platinum Taq DNA Polymerase High Fidelity (Thermo Scientific). The PCRs were carried out under the following conditions: preheating $95^{\circ} \mathrm{C}$ for $5 \mathrm{~min}$, followed by 35 cycles of $95^{\circ} \mathrm{C}$ for $30 \mathrm{~s}, 64^{\circ} \mathrm{C}$ for $30 \mathrm{~s}$, and $68^{\circ} \mathrm{C}$ for $1 \mathrm{~min} 20 \mathrm{~s}$.

The PCR products were resolved on $1.5 \%$ agarose gel and then purified with Qiaquick PCR Purification Kit (QIAGEN). For T7E1 cleavage assay purified PCR products were denatured and annealed in NEBuffer 2 (NEB) using a thermocycler. Hybridized PCR products were digested with $\mathrm{T} 7$ endonuclease 1 (NEB, M0302L) for $15 \mathrm{~min}$ at $37^{\circ} \mathrm{C}$ and subjected to $2 \%$ agarose gel electrophoresis. Percentage of DNA modification was calculated using the formula \%DNA modification $=100^{\star}\left(1-(1-\text { fraction cleaved })^{1 / 2}\right)$ reported on the NEB website (https://www.neb.com/protocols/2014/08/11/determining-genometargeting-efficiency-using-t7-endonuclease-i). All PCR primer sequences are listed in Table S2.

\section{Detection of Deletions and Inversions}

The gDNA samples obtained from cells transfected with two gRNAs were amplified with Platinum Taq DNA Polymerase High Fidelity (Thermo Scientific). We prepared two specific PCRs with a common forward primer upstream the first gRNA binding site and a second 
forward primer localized between the two gRNA binding sites to detect inversions or a reverse primer downstream the second gRNA binding site to detect inversions (Figures 2C and 2D). The PCRs were conducted in order to detect genomic deletions and inversions under these conditions: preheating $95^{\circ} \mathrm{C}$ for $5 \mathrm{~min}$, followed by $35 \mathrm{cy}-$ cles of $95^{\circ} \mathrm{C}$ for $30 \mathrm{~s}, 60^{\circ} \mathrm{C}$ for $30 \mathrm{~s}$, and $68^{\circ} \mathrm{C}$ for $40 \mathrm{~s}$. Inversions were amplified with 40 cycles.

Inversions in cells with duplication of the exon 2 were amplified with only one forward primer upstream of the binding site of the gRNA used. If inversions occur, the primer would bind to the wild-type sequence in forward orientation and the inverted one in reverse orientation (Figure S3). PCR conditions were preheating $95^{\circ} \mathrm{C}$ for $5 \mathrm{~min}$, followed by 45 cycles of $95^{\circ} \mathrm{C}$ for $30 \mathrm{~s}, 60^{\circ} \mathrm{C}$ for $30 \mathrm{~s}$, and $68^{\circ} \mathrm{C}$ for $40 \mathrm{~s}$.

Amplicons were resolved through 1.5\% agarose gel electrophoresis. All PCR primer sequences are listed in Table S3.

\section{Lentivirus Production, Titration, and Transduction}

In order to increase the efficacy and maximize our results in cell lines, we cloned selected gRNAs in the LentiCRISPRv2 vector (Addgene Plasmid \#52961). ${ }^{35}$

\section{Research Grade Lentiviral Vector Production}

Third-generation lentiviral vectors were produced by calcium phosphate transient transfection of 293T cells of the selected transfer vector, the packaging plasmid pKLg/p.RRE, pK.REV, the vesicular stomatitis virus glycoprotein $\mathrm{G}$ (VSV-G) envelope plasmid pK.G as previously described. ${ }^{49}$

\section{Lentiviral Vector Titration}

Three hundred thousand $\left(3 \times 10^{5}\right)$ HCT116 cells were transduced with serial vector dilutions in the presence of polybrene $(6 \mu \mathrm{g} / \mathrm{mL})$. Genomic DNA (gDNA) was extracted $72 \mathrm{hr}$ after transduction. gDNA is extracted by using MagNaPure 96 system (Roche Diagnostics, Promega) according to manufacturer's instructions. Vector copies per diploid genome (VCN) were quantified by quantitative PCR (qPCR) starting from $100 \mathrm{ng}$ of template gDNA using primers (HIV sense: 5'-TACTGACGCTCTCGCACC-3'; HIV antisense: $5^{\prime}$-TCTCGACGCAGGACTCG- $3^{\prime}$ ) and a probe FAM $5^{\prime}$ - ATCTCTC TCCTTCTAGCCTC-3') against the packaging signal region of LVs. Endogenous DNA amount was quantified by a primers/probe set against the human albumin gene (ALB sense: $5^{\prime}$ GCTGTCATCT CTTGTGGGCTGT-3'; ALB antisense: $5^{\prime}$-ACTCATGGGAGCTGC TGGTTC-3'; ALB probe: VIC 5'-CCTGTCATGCCCACACAAAT CTCTCC- $\left.3^{\prime}\right)$. Copies per genome were calculated by the formula $=$ [ng LV/ng endogenous DNA] $\times$ [no. of LV integrations in the standard curve]. The standard curve was generated by using a plasmid containing the appropriate sequences in cis from the vectors and ALB gene. Vector particles were measured by HIV-1 Gag p24 antigen immunocapture assay (PerkinElmer) according to manufacturer's instructions. Vector infectivity is calculated as the ratio between titer and particles. ${ }^{50}$
Titers obtained ranged from 5.8E8 transducing units (TU)/mL to 1.1E9 TU/mL. LV_int2.1, LV_int2.6 and LV_ex2.1 were used at MOI 20 together with $8 \mu \mathrm{g} / \mathrm{mL}$ of polybrene in exon 2 duplicated myoblasts cell lines.

\section{Quantification of Wild-Type Alleles Created after Removal of the Exon 2 Duplication by One gRNA}

Quantification of the efficacy of the gRNAs to remove the duplicated sequence at the DNA level was performed by Real-Time PCR using SsoFast EvaGreen Supermix (Bio-Rad) for 40 cycles and primers designed on the minimal common duplicated region (forward CCAAACCCATAGTCGTTCAAAT, reverse AGATGCTGCAAA GGAAGGAA) and on intron 67 of the DMD gene as control (forward GCATTTGGAAAGATTTGCTGA, reverse TGCTGGTGC AGACTTCTCTC). Real-Time PCR was performed in triplicate in 96-well plates using $50 \mathrm{ng}$ genomic DNA from wild-type, untreated, and lentiviral transduced cells. The calculation of the fold changes was based on the $2-\Delta \Delta C \mathrm{CT}$ method (Applied Biosystems User Bulletin \#2) and results were expressed in relation to the untreated cells set to 1 .

\section{RNA Analysis}

Total RNA was isolated from myotubes three days after differentiation using the RNeasy Kit (QIAGEN) and reverse-transcribed by means of a High-Capacity cDNA Reverse Transcription Kit (Applied Biosystems), according to the manufacturer's instructions.

Before cDNA synthesis, RNA was treated with DNase I (Roche) and checked for residual DNA contamination by a 55-cycle PCR.

RT-PCR of the exon 2 duplication was performed with Platinum Taq DNA Polymerase High Fidelity (Thermo Scientific) and oligos 21F and 448R (designed complementary to exon 1 and 4 of the DMD muscle isoform and taken from http://dmd.nl/) and resolved on $2 \%$ agarose gel. One microliter of the RT-PCR was loaded on high-sensitivity DNA chips (Agilent) for the quantification of the duplication excision, which was performed calculating the ratio of the area of the normal transcript and the sum of the area of normal and duplicated transcripts multiplied for 100 and normalized by setting to one the untreated (UT) samples. Experiments were performed in triplicate, mean results and SDs are shown. Significance was evaluated by Kruskal-Wallis test with post hoc Mann-Whitney test for $\mathrm{p}$ values less than 0.05 considered significant.

\section{Western Blot and Immunofluorescence Analysis}

Immortalized myoblasts seeded in 30-cm Petri dishes and differentiated for 10 days were washed with cold PBS and lysed in radioimmunoprecipitation assay (RIPA) buffer in presence of Complete Mini protease inhibitor mixture (Roche) for $30 \mathrm{~min}$ on ice. Cell lysates were cleared and quantified.

To check the expression of dystrophin, $30 \mu \mathrm{g}$ of protein from myotubes was mixed with $4 \times$ NuPage LDS buffer (Invitrogen) supplemented with $50 \mathrm{mM}$ DTT, heated for $2 \mathrm{~min}$ at $85^{\circ} \mathrm{C}$, loaded on 
Novex 3\%-8\% Tris-Acetate Midi Gel (Invitrogen), and electrophoresed at $150 \mathrm{~V}$ with Tris-Acetate SDS running buffer (Invitrogen) for $70 \mathrm{~min}$ at room temperature. Proteins were then transferred at $45 \mathrm{~V}$ overnight at $4^{\circ} \mathrm{C}$ onto polyvinylidene fluoride (PVDF) membranes as described above using transfer buffer with no methanol.

Membranes were blocked in Tris-buffered saline-tween (TBS-T) (20 mM Tris, $500 \mathrm{mM} \mathrm{NaCl}[\mathrm{pH}=7.5], 0.1 \%$ Tween 20) supplemented with $3 \%$ (Dystrophin and Cas9) or 5\% ( $\alpha$-actinin) non-fat dry milk (Bio-Rad) for $1 \mathrm{hr}$ at room temperature.

Membranes were incubated for $1 \mathrm{hr}$ at room temperature with rabbit polyclonal anti-Dystrophin (ab15266, Abcam), anti-CRISPR-Cas9 (ab191468, Abcam), and mouse monoclonal $\alpha$-actinin (Santa Cruz Biotechnology) antibodies, washed three times for $10 \mathrm{~min}$ in TBS-T, incubated with immunopure goat anti-rabbit or rabbit anti-mouse immunoglobulin Gs (IgGs) (Dako) conjugated to horseradish peroxidase (HRP, 1:2,000 in TBS-T 3 or 5\% milk) for $1 \mathrm{hr}$ at room temperature, and revealed with Pierce ECL Western Blotting Substrate (Pierce Biotechnology). Chemiluminescence was acquired by the ChemiDoc MP System (Bio-Rad) and quantified using ImageJ v.1.37 (NIH image). Immortalized control myoblasts differentiated into myotubes in vitro were used as positive control.

In vitro differentiated immortalized wild-type, \#515 and \#994 myoblasts were washed with PBS (Sigma-Aldrich), fixed with $4 \%$ paraformaldehyde (Sigma-Aldrich) at room temperature for $10 \mathrm{~min}$, and permeabilized with ethanol $75 \%$ (Sigma-Aldrich) at room temperature for $1 \mathrm{~min}$. As a blocking solution, 20\% fetal bovine serum (Sigma-Aldrich) was used at room temperature for $30 \mathrm{~min}$ to reduce secondary antibody background signal. The cells were subsequently incubated overnight at $4^{\circ} \mathrm{C}$ with the anti-dystrophin (NCL-DYS2 Novocastra) primary antibody. After incubation, cells were washed with PBS and then incubated with the 594-fluorochrome conjugated secondary antibodies (Thermo Fisher) together with DAPI for nucleic acid staining (Ibidi) for $1 \mathrm{hr}$ at room temperature in PBS containing $0.2 \%$ Triton X-100. After three successive washings with PBS, dishes were mounted using fluorescent mounting medium (Ibidi) and examined by fluorescence microscopy (DMI6000B, Leica).

\section{SUPPLEMENTAL INFORMATION}

Supplemental Information includes four figures and three tables and can be found with this article online at http://dx.doi.org/10.1016/j. omtn.2017.02.004.

\section{AUTHOR CONTRIBUTIONS}

Conceptualization, M.B.; Methodology, M.B.; Investigation, A.L., S.D., A.M., A.I., and E.B.; Writing - Original Draft, M.B. and A.L.; Writing - Review \& Editing, V.M., F.B., F.M., and M.B.; Funding Acquisition, F.M. and M.B.; Resources, S.M., K.M., and V.M.; Supervision, F.M. and M.B.

\section{CONFLICTS OF INTEREST}

We declare no competing financial interests.

\section{ACKNOWLEDGMENTS}

France Leturcq (Laboratoire de biochimie et génétique, moléculaire, Hôpital Cochin et Institut de Myologie, Groupe hospitalier La Pitié Salpétrière, APHP, France) and AFM-Telethon Biobank are gratefully acknowledged for providing patients samples and cells. Plasmids MLM3636 (Addgene plasmid \# 43860), JDS246 (\#43861), and LentiCRISPRv2 (\#52961) were a gift from Keith Joung and Feng Zhang. This work was supported by grants from the European Research Council (ERC-2010-AdG, GT-SKIN), the association "Amici di Edy," AFM-Téléthon (Trampoline grant no. 17247), and Horinzon 2020 Marie Sklodowska-Curie Individual Fellowship Action (Project DMD2CURE \#703093) to M.B. Federica Brugnoli (Section of Anatomy and Histology, Department of Morphology, Surgery and Experimental Medicine, University of Ferrara, Ferrara, Italy) is also acknowledged for the technical support.

\section{REFERENCES}

1. Mendell, J.R., Shilling, C., Leslie, N.D., Flanigan, K.M., al-Dahhak, R., Gastier-Foster, J., Kneile, K., Dunn, D.M., Duval, B., Aoyagi, A., et al. (2012). Evidence-based path to newborn screening for Duchenne muscular dystrophy. Ann. Neurol. 71, 304-313.

2. Kinali, M., Arechavala-Gomeza, V., Cirak, S., Glover, A., Guglieri, M., Feng, L., Hollingsworth, K.G., Hunt, D., Jungbluth, H., Roper, H.P., et al. (2011). Muscle histology vs MRI in Duchenne muscular dystrophy. Neurology 76, 346-353.

3. Aartsma-Rus, A., Van Deutekom, J.C., Fokkema, I.F., Van Ommen, G.J., and Den Dunnen, J.T. (2006). Entries in the Leiden Duchenne muscular dystrophy mutation database: An overview of mutation types and paradoxical cases that confirm the reading-frame rule. Muscle Nerve 34, 135-144.

4. Wilton, S.D., Veedu, R.N., and Fletcher, S. (2015). The emperor's new dystrophin Finding sense in the noise. Trends Mol. Med. 21, 417-426.

5. Lu, Q.L., Cirak, S., and Partridge, T. (2014). What can we learn from clinical trials of exon skipping for DMD? Mol. Ther. Nucleic Acids 3, e152.

6. Aoki, Y., Nakamura, A., Yokota, T., Saito, T., Okazawa, H., Nagata, T., and Takeda, S. (2010). In-frame dystrophin following exon 51-skipping improves muscle pathology and function in the exon 52-deficient mdx mouse. Mol. Ther. 18, 1995-2005.

7. Arechavala-Gomeza, V., Graham, I.R., Popplewell, L.J., Adams, A.M., Aartsma-Rus, A., Kinali, M., Morgan, J.E., van Deutekom, J.C., Wilton, S.D., Dickson, G., and Muntoni, F. (2007). Comparative analysis of antisense oligonucleotide sequences for targeted skipping of exon 51 during dystrophin pre-mRNA splicing in human muscle. Hum. Gene Ther. 18, 798-810.

8. Aartsma-Rus, A., Janson, A.A., van Ommen, G.J., and van Deutekom, J.C. (2007). Antisense-induced exon skipping for duplications in Duchenne muscular dystrophy. BMC Med. Genet. 8, 43.

9. Greer, K.L., Lochmüller, H., Flanigan, K., Fletcher, S., and Wilton, S.D. (2014). Targeted exon skipping to correct exon duplications in the dystrophin gene. Mol. Ther. Nucleic Acids 3, e155.

10. van Vliet, L., de Winter, C.L., van Deutekom, J.C., van Ommen, G.J., and AartsmaRus, A. (2008). Assessment of the feasibility of exon 45-55 multiexon skipping for Duchenne muscular dystrophy. BMC Med. Genet. 9, 105.

11. Taylor, P.J., Maroulis, S., Mullan, G.L., Pedersen, R.L., Baumli, A., Elakis, G., Piras, S., Walsh, C., Prósper-Gutiérrez, B., De La Puente-Alonso, F., et al. (2007). Measurement of the clinical utility of a combined mutation detection protocol in carriers of Duchenne and Becker muscular dystrophy. J. Med. Genet. 44, 368-372.

12. Wein, N., Vulin, A., Falzarano, M.S., Szigyarto, C.A., Maiti, B., Findlay, A., Heller, K.N., Uhlén, M., Bakthavachalu, B., Messina, S., et al. (2014). Translation from a DMD exon 5 IRES results in a functional dystrophin isoform that attenuates dystrophinopathy in humans and mice. Nat. Med. 20, 992-1000.

13. Ryan, N.J. (2014). Ataluren: First global approval. Drugs 74, 1709-1714.

14. Traynor, K. (2016). Eteplirsen approved for Duchenne muscular dystrophy. Am. J. Health Syst. Pharm. 73, 1719. 
15. Gaj, T., Gersbach, C.A., and Barbas, C.F., 3rd (2013). ZFN, TALEN, and CRISPR/ Cas-based methods for genome engineering. Trends Biotechnol. 31, 397-405.

16. Joung, J.K., and Sander, J.D. (2013). TALENs: A widely applicable technology for targeted genome editing. Nat. Rev. Mol. Cell Biol. 14, 49-55.

17. Silva, G., Poirot, L., Galetto, R., Smith, J., Montoya, G., Duchateau, P., and Pâques, F. (2011). Meganucleases and other tools for targeted genome engineering: Perspectives and challenges for gene therapy. Curr. Gene Ther. 11, 11-27.

18. Mali, P., Yang, L., Esvelt, K.M., Aach, J., Guell, M., DiCarlo, J.E., Norville, J.E., and Church, G.M. (2013). RNA-guided human genome engineering via Cas9. Science $339,823-826$.

19. Nakamura, K., Fujii, W., Tsuboi, M., Tanihata, J., Teramoto, N., Takeuchi, S., Naito, K., Yamanouchi, K., and Nishihara, M. (2014). Generation of muscular dystrophy model rats with a CRISPR/Cas system. Sci. Rep. 4, 5635.

20. Chen, Y., Zheng, Y., Kang, Y., Yang, W., Niu, Y., Guo, X., Tu, Z., Si, C., Wang, H., Xing, R., et al. (2015). Functional disruption of the dystrophin gene in rhesus monkey using CRISPR/Cas9. Hum. Mol. Genet. 24, 3764-3774.

21. Larcher, T., Lafoux, A., Tesson, L., Remy, S., Thepenier, V., François, V., Le Guiner, C., Goubin, H., Dutilleul, M., Guigand, L., et al. (2014). Characterization of dystrophin deficient rats: A new model for Duchenne muscular dystrophy. PLoS ONE 9, e110371.

22. Ousterout, D.G., Perez-Pinera, P., Thakore, P.I., Kabadi, A.M., Brown, M.T., Qin, X., Fedrigo, O., Mouly, V., Tremblay, J.P., and Gersbach, C.A. (2013). Reading frame correction by targeted genome editing restores dystrophin expression in cells from Duchenne muscular dystrophy patients. Mol. Ther. 21, 1718-1726.

23. Ousterout, D.G., Kabadi, A.M., Thakore, P.I., Perez-Pinera, P., Brown, M.T., Majoros, W.H., Reddy, T.E., and Gersbach, C.A. (2015). Correction of dystrophin expression in cells from Duchenne muscular dystrophy patients through genomic excision of exon 51 by zinc finger nucleases. Mol. Ther. 23, 523-532.

24. Li, H.L., Fujimoto, N., Sasakawa, N., Shirai, S., Ohkame, T., Sakuma, T., Tanaka, M. Amano, N., Watanabe, A., Sakurai, H., et al. (2015). Precise correction of the dystrophin gene in duchenne muscular dystrophy patient induced pluripotent stem cells by TALEN and CRISPR-Cas9. Stem Cell Reports 4, 143-154.

25. Long, C., McAnally, J.R., Shelton, J.M., Mireault, A.A., Bassel-Duby, R., and Olson, E.N. (2014). Prevention of muscular dystrophy in mice by CRISPR/Cas9-mediated editing of germline DNA. Science 345, 1184-1188.

26. Goyenvalle, A., Griffith, G., Babbs, A., El Andaloussi, S., Ezzat, K., Avril, A., Dugovic, B., Chaussenot, R., Ferry, A., Voit, T., et al. (2015). Functional correction in mouse models of muscular dystrophy using exon-skipping tricyclo-DNA oligomers. Nat. Med. 21, 270-275.

27. Vila, M.C., Klimek, M.B., Novak, J.S., Rayavarapu, S., Uaesoontrachoon, K., Boehler, J.F., Fiorillo, A.A., Hogarth, M.W., Zhang, A., Shaughnessy, C., et al. (2015). Elusive sources of variability of dystrophin rescue by exon skipping. Skelet. Muscle 5, 44.

28. Wojtal, D., Kemaladewi, D.U., Malam, Z., Abdullah, S., Wong, T.W., Hyatt, E., Baghestani, Z., Pereira, S., Stavropoulos, J., Mouly, V., et al. (2016). Spell checking nature: Versatility of CRISPR/Cas9 for developing treatments for inherited disorders. Am. J. Hum. Genet. 98, 90-101.

29. Bovolenta, M., Neri, M., Fini, S., Fabris, M., Trabanelli, C., Venturoli, A., Martoni, E. Bassi, E., Spitali, P., Brioschi, S., et al. (2008). A novel custom high density-comparative genomic hybridization array detects common rearrangements as well as deep intronic mutations in dystrophinopathies. BMC Genomics 9, 572.

30. Bovolenta, M., Scotton, C., Falzarano, M.S., Gualandi, F., and Ferlini, A. (2012). Rapid, comprehensive analysis of the dystrophin transcript by a custom micro-fluidic exome array. Hum. Mutat. 33, 572-581.

31. del Gaudio, D., Yang, Y., Boggs, B.A., Schmitt, E.S., Lee, J.A., Sahoo, T., Pham, H.T., Wiszniewska, J., Chinault, A.C., Beaudet, A.L., and Eng, C.M. (2008). Molecular diagnosis of Duchenne/Becker muscular dystrophy: Enhanced detection of dystrophin gene rearrangements by oligonucleotide array-comparative genomic hybridization. Hum. Mutat. 29, 1100-1107.

32. White, S.J., Aartsma-Rus, A., Flanigan, K.M., Weiss, R.B., Kneppers, A.L., Lalic, T., Janson, A.A., Ginjaar, H.B., Breuning, M.H., and den Dunnen, J.T. (2006). Duplications in the DMD gene. Hum. Mutat. 27, 938-945.
33. Mamchaoui, K., Trollet, C., Bigot, A., Negroni, E., Chaouch, S., Wolff, A., Kandalla, P.K., Marie, S., Di Santo, J., St Guily, J.L., et al. (2011). Immortalized pathological human myoblasts: Towards a universal tool for the study of neuromuscular disorders. Skelet. Muscle 1, 34

34. Kraft, K., Geuer, S., Will, A.J., Chan, W.L., Paliou, C., Borschiwer, M., Harabula, I., Wittler, L., Franke, M., Ibrahim, D.M., et al. (2015). Deletions, inversions, duplications: Engineering of structural variants using CRISPR/Cas in mice. Cell Rep. 10, 833-839.

35. Sanjana, N.E., Shalem, O., and Zhang, F. (2014). Improved vectors and genome-wide libraries for CRISPR screening. Nat. Methods 11, 783-784.

36. Ousterout, D.G., Kabadi, A.M., Thakore, P.I., Majoros, W.H., Reddy, T.E., and Gersbach, C.A. (2015). Multiplex CRISPR/Cas9-based genome editing for correction of dystrophin mutations that cause Duchenne muscular dystrophy. Nat. Commun. 6, 6244.

37. Tabebordbar, M., Zhu, K., Cheng, J.K., Chew, W.L., Widrick, J.J., Yan, W.X. Maesner, C., Wu, E.Y., Xiao, R., Ran, F.A., et al. (2016). In vivo gene editing in dystrophic mouse muscle and muscle stem cells. Science 351, 407-411.

38. Nelson, C.E., Hakim, C.H., Ousterout, D.G., Thakore, P.I., Moreb, E.A., Castellanos Rivera, R.M., Madhavan, S., Pan, X., Ran, F.A., Yan, W.X., et al. (2016). In vivo genome editing improves muscle function in a mouse model of Duchenne muscular dystrophy. Science 351, 403-407.

39. Long, C., Amoasii, L., Mireault, A.A., McAnally, J.R., Li, H., Sanchez-Ortiz, E. Bhattacharyya, S., Shelton, J.M., Bassel-Duby, R., and Olson, E.N. (2016). Postnatal genome editing partially restores dystrophin expression in a mouse model of muscular dystrophy. Science 351, 400-403.

40. Xu, L., Park, K.H., Zhao, L., Xu, J., El Refaey, M., Gao, Y., Zhu, H., Ma, J., and Han, R. (2016). CRISPR-mediated Genome Editing Restores Dystrophin Expression and Function in mdx Mice. Mol. Ther. 24, 564-569.

41. Lu, Q.L., Mann, C.J., Lou, F., Bou-Gharios, G., Morris, G.E., Xue, S.A., Fletcher, S., Partridge, T.A., and Wilton, S.D. (2003). Functional amounts of dystrophin produced by skipping the mutated exon in the mdx dystrophic mouse. Nat. Med. 9, 1009-1014.

42. Doench, J.G., Fusi, N., Sullender, M., Hegde, M., Vaimberg, E.W., Donovan, K.F., Smith, I., Tothova, Z., Wilen, C., Orchard, R., et al. (2016). Optimized sgRNA design to maximize activity and minimize off-target effects of CRISPR-Cas9. Nat. Biotechnol. 34, 184-191.

43. Kleinstiver, B.P., Pattanayak, V., Prew, M.S., Tsai, S.Q., Nguyen, N.T., Zheng, Z., and Joung, J.K. (2016). High-fidelity CRISPR-Cas9 nucleases with no detectable genomewide off-target effects. Nature 529, 490-495.

44. Ran, F.A., Cong, L., Yan, W.X., Scott, D.A., Gootenberg, J.S., Kriz, A.J., Zetsche, B. Shalem, O., Wu, X., Makarova, K.S., et al. (2015). In vivo genome editing using Staphylococcus aureus Cas9. Nature 520, 186-191.

45. Zetsche, B., Gootenberg, J.S., Abudayyeh, O.O., Slaymaker, I.M., Makarova, K.S., Essletzbichler, P., Volz, S.E., Joung, J., van der Oost, J., Regev, A., et al. (2015). Cpf1 is a single RNA-guided endonuclease of a class 2 CRISPR-Cas system. Cell $163,759-771$.

46. Le Hir, M., Goyenvalle, A., Peccate, C., Précigout, G., Davies, K.E., Voit, T., Garcia, L. and Lorain, S. (2013). AAV genome loss from dystrophic mouse muscles during AAV-U7 snRNA-mediated exon-skipping therapy. Mol. Ther. 21, 1551-1558.

47. Fu, Y., Reyon, D., and Joung, J.K. (2014). Targeted genome editing in human cells using CRISPR/Cas nucleases and truncated guide RNAs. Methods Enzymol. 546, $21-45$.

48. Wang, T., Wei, J.J., Sabatini, D.M., and Lander, E.S. (2014). Genetic screens in human cells using the CRISPR-Cas9 system. Science 343, 80-84.

49. Cantore, A., Ranzani, M., Bartholomae, C.C., Volpin, M., Valle, P.D., Sanvito, F., Sergi, L.S., Gallina, P., Benedicenti, F., Bellinger, D., et al. (2015). Liver-directed lentiviral gene therapy in a dog model of hemophilia B. Sci. Transl. Med. 7, $277 \mathrm{ra} 28$.

50. Zanta-Boussif, M.A., Charrier, S., Brice-Ouzet, A., Martin, S., Opolon, P., Thrasher, A.J., Hope, T.J., and Galy, A. (2009). Validation of a mutated PRE sequence allowing high and sustained transgene expression while abrogating WHV-X protein synthesis: Application to the gene therapy of WAS. Gene Ther. 16, 605-619. 
OMTN, Volume 7

\section{Supplemental Information}

\section{Correction of the Exon 2 Duplication in DMD}

\section{Myoblasts by a Single CRISPR/Cas9 System}

Annalisa Lattanzi, Stephanie Duguez, Arianna Moiani, Araksya Izmiryan, Elena Barbon, Samia Martin, Kamel Mamchaoui, Vincent Mouly, Francesco Bernardi, Fulvio Mavilio, and Matteo Bovolenta 


\section{SUPPLEMENTARY FIGURES AND TABLES}

a

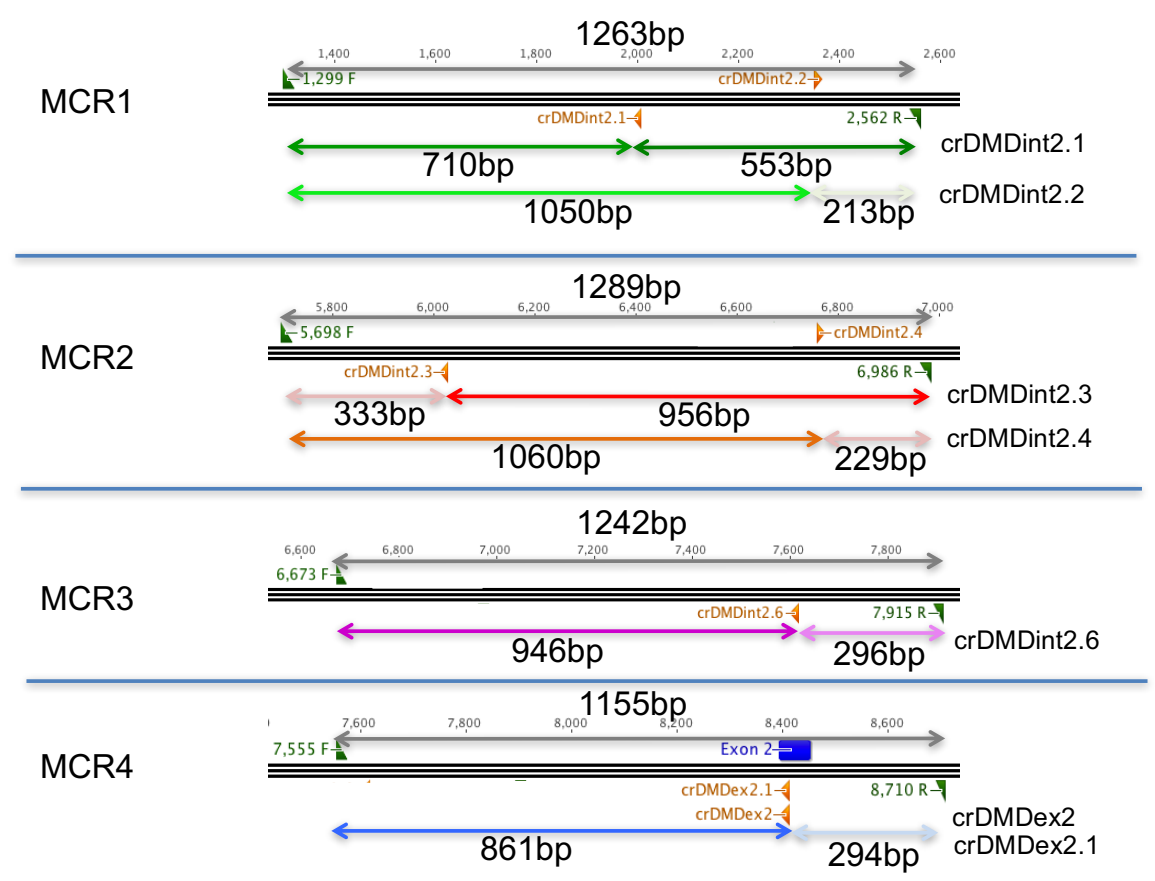

b

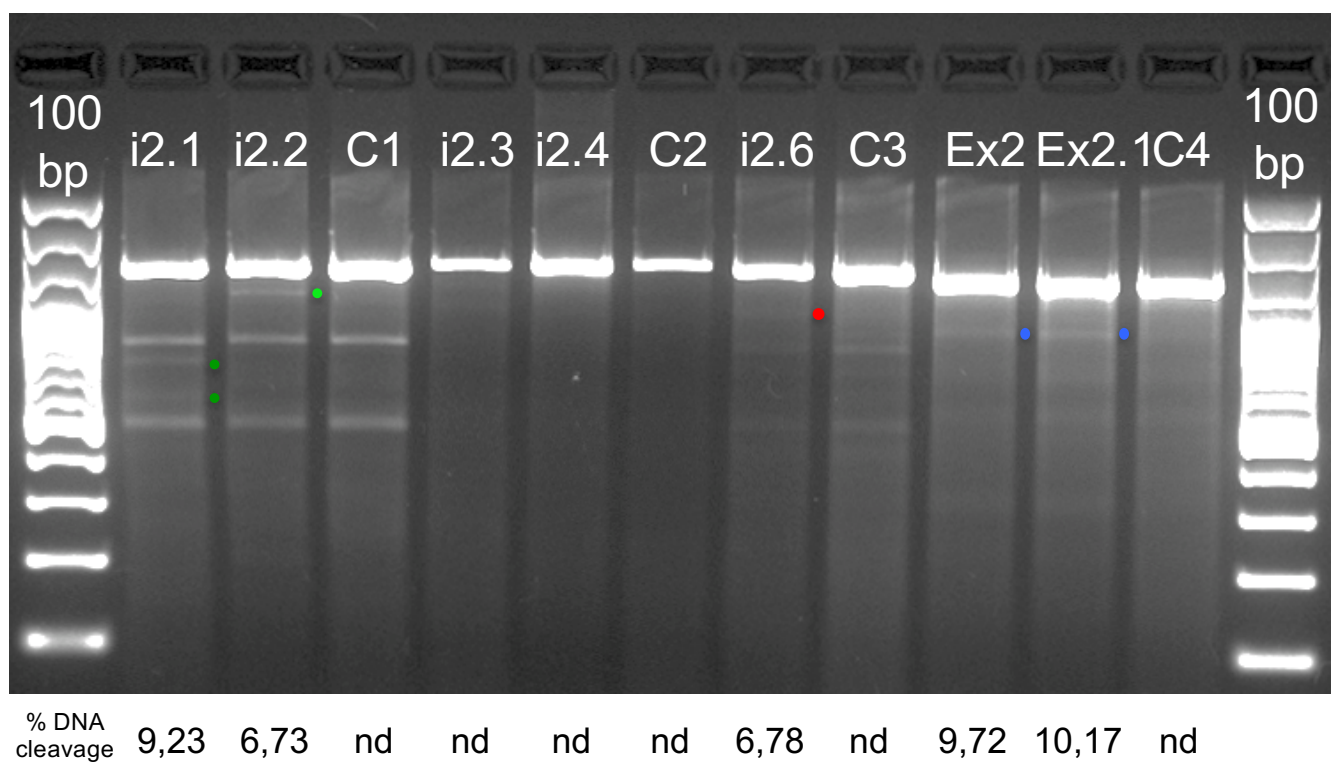

Figure S1. Testing gRNAs for NHEJ indel formation in HEK293T cells: (a) schematic of the PCR systems designed to amplify four regions of the minimal common duplicated region (MCR). For each region the intervening gRNAs are shown and predicted fragments generated by the T7E1 treatment are color-coded. (b) T7E1 assay of PCR products from HEK293T cells transfected with MLM3636 gRNA plasmids and spCas9 (JDS246). Coloured Dots indicate the position of T7E1 digestion fragments related to the activity of the gRNAs. Percentage of DNA cleavage is reported below each lane. 


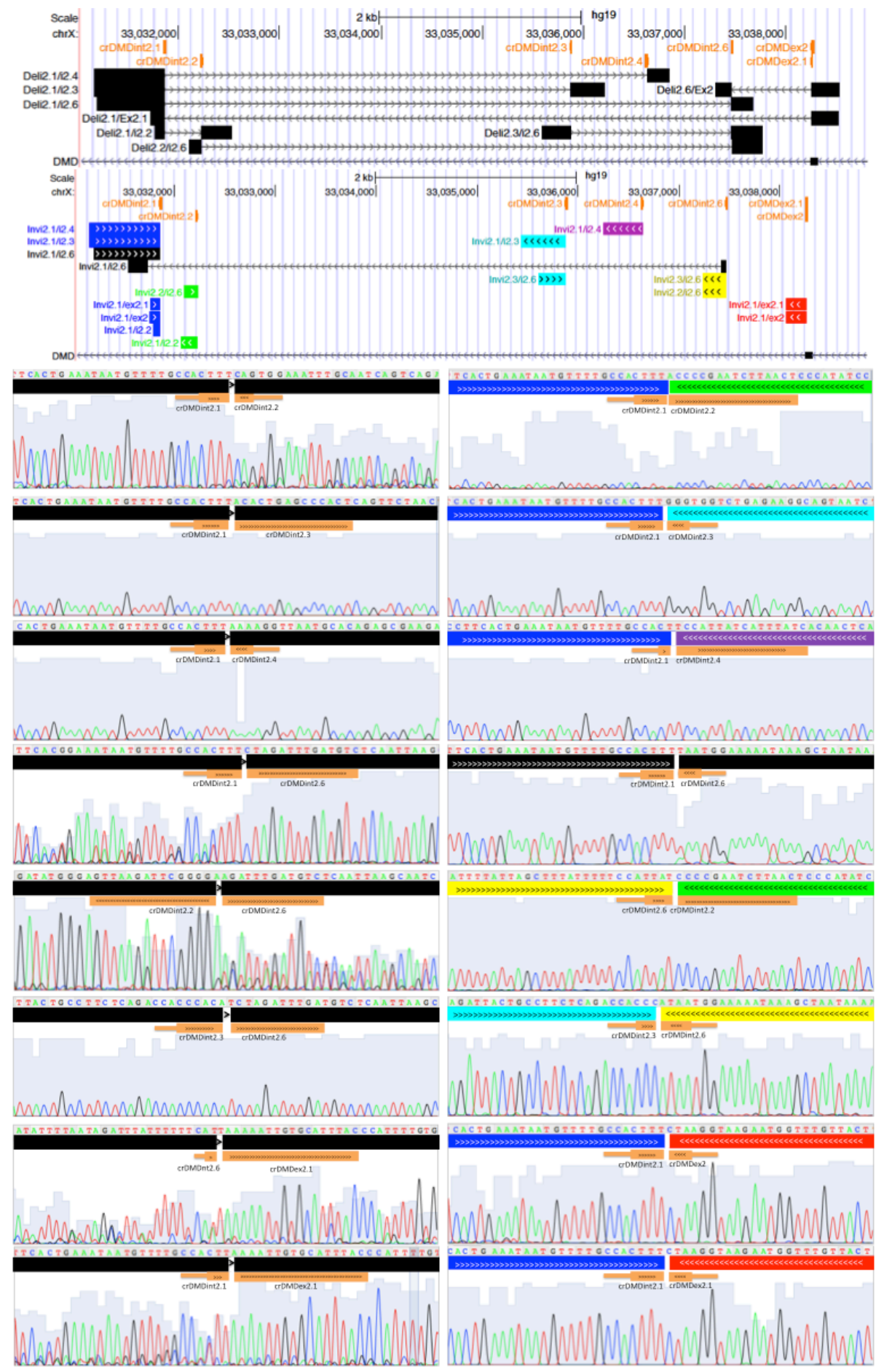

Figure S2: Characterization of deletion and Inversion Breakpoints.

Visualization of deletions and inversions caused by the activity of two gRNAS obtained by loading the resulting sequences (left panels: deletions; right panels: inversions) in UCSC Genome Browser and creating two custom tracks.

The sequences of each gRNA are overlaid over the chromatograms and represented by an orange thick bar for the PAM sequence and arrowed orange bars for the binding sequences. Deletion breakpoints are represented as black arrows between two black bars whereas inversion is shown as colour-coded bars with arrows indicating the original orientation of the sequence. 

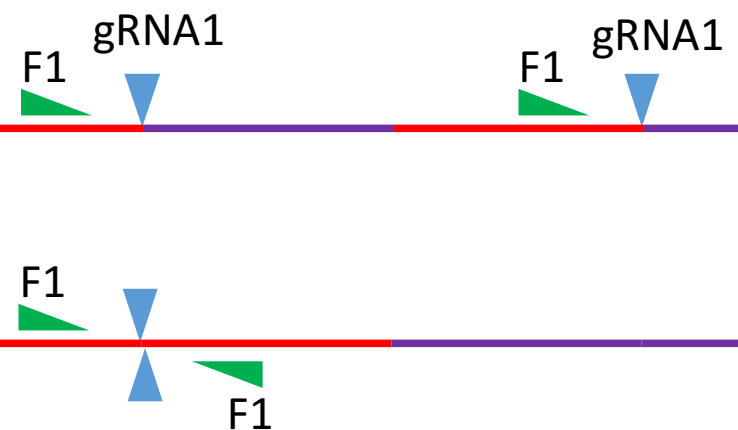

Figure S3: Strategy to amplify inversions of the duplicated region caused by activity of a gRNA with one primer. The primer and the gRNA are represented as green and light blue triangles respectively. The tandem duplication is coloured in red and violet in order to easily recognise the inversion in the scheme. 
Deletions PAM gRNA Int2.1

TACCTCCTTCACTGAAATAATGTTTTGCCACTTTGTCCCAGTATCATCCTTTTTTAAAAACTTCTAAGCTCATTTTAAAT WT 26.3(29/110)

TACCTCCTTCACTGAAATAAGTTTTGCCACTT-GTCCCAGTATCATCCTTTTTTAAAAACTTCTAAGCTCATTTTAAAT x13 TACCTCCTTCACTGAAATAATGTTTTGCCACTTT--CCCAGTATCATCCTTTTTTAAAAACTTCTAAGCTCATTTTAAAT x8 TACCTCCTTCACTGAAATAATGTTTGCCACT----CCCAGTATCATCCTTTTTTAAAAACTTCTAAGCTCATTTTAAAT x3 TACCTCCTTCACTGAAATAATGTTTTGCCACTT-----CAGTATCATCCTTTTTTAAAAACTTCTAAGCTCATTTTAAAT x2 TACCTCCTTCACTGAAATAATGTTTTGCCACTTT---CCAGTATCATCCTTTTTTAAAAACTTCTAAGCTCATTTTAAAT TACCTCCTTCACTGAAATAATGTTTGCCACTTT----CAGTATCATCCTTTTTTAAAAACTTCTAAGCTCATTTTAAAT TACCTCCTTCACTGAAATAATGTTTTGCCACTT---CCCAGTATCATCCTTTTTTAAAAACTTCTAAGCTCATTTTAAAT TACCTCCTTCACTGAAATAATGTTTTGCCACTTT-----AGTATCATCCTTTTTTAAAAACTTCTAAGCTCATTTTAAAT TACCTCCTTCACTGAAATAATGTTTTGCCACTT--------ATCATCCTTTTTTAAAAACTTCTAAGCTCATTTTAAAT TACCTCCTTCACTGAAATAATGTTTGCCACTTT---------------TTTAAAAACTTCTAAGCTCATTTTAAAT

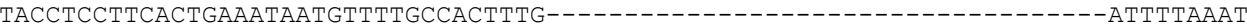
TACCTCCTTCACTGAAATAATGTTTTGCCACTTT--------(133)--------------ATGTTTACTGGGTGTG

Deletions: $\quad 30.9 \%(34 / 110)$

Insertions

TACCTCCTTCACTGAAATAATGTTTGCCACTTT---GT-CCCAGTATCATCCTTTTTTAAAAACTTCTAAGCTCATTTTAAAT WT

TACCTCCTTCACTGAAATAATGTTTGCCACTTT--TGT-CCCAGTATCATCCTTTTTTAAAAACTTCTAAGCTCATTTTAAAT insT x41 TACCTCCTTCACTGAAATAATGTTTTGCCACTTT-ATGT-CCCAGTATCATCCTTTTTTAAAAACTTCTAAGCTCATTTTAAAT insAT х2 TACCTCCTTCACTGAAATAATGTTTTGCCACTTTTATGT-CCCAGTATCATCCTTTTTTAAAAACTTCTAAGCTCATTTTAAAT insTAT TACCTCCTTCACTGAAATAATGTTTGCCACTTTCTTGT-CCCAGTATCATCCTTTTTTAAAAACTTCTAAGCTCATTTTAAAT insCTT TACCTCCTTCACTGAAATAATGTTTGCCACTTT---GTGCCCAGTATCATCCTTTTTTAAAAACTTCTAAGCTCATTTTAAAT insG

$$
\text { Insertions: } \quad 41.8 \%(46 / 110)
$$

Indels

TACCTCCTTCACTGAAATAATGTTTGCCACTTT---C--CCCAGTATCATCCTTTTTTAAAAACTTCTAAGCTCATTTTAAAT insC delGT

$$
\text { Indels: } \quad 0.01 \%(1 / 110)
$$

\begin{tabular}{|c|c|c|c|c|c|c|c|c|c|}
\hline Potential off-target site \# & Sequence & Indels (\%) & Chromosome \# & Strand & Position & GENE & Mismatch \#1 & Mismatch \#2 & Mismatch \#3 \\
\hline Site Of Interest & GATGATACTGGGACAAAG & $72.71 \%$ & & & & DMD, intronic & & & \\
\hline Potential Off-Target site \# 1 & GTTGAAACTGGGACAAAG & nd & chr6 & - & 105368929 & none & $1: A>T$ & $5: T>A$ & \\
\hline Potential Off-Target site \# 2 & GAAGATACTGGGAGAAAG & nd & chr2 & + & 217969247 & none & $2: A>T$ & $13: G>C$ & \\
\hline Potential Off-Target site \# 3 & GATGAGACTGGGAGAAAG & nd & chr12 & + & 90417929 & none & $5: G>T$ & $13: G>C$ & \\
\hline 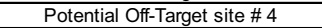 & GATGACAGTGGGACAAAG & nd & chr2 & - & 11851832 & $\begin{array}{l}\text { LPIN1, intronic } \\
\end{array}$ & $5: G>A$ & $7: C>G$ & \\
\hline Potential Off-Target site \# 6 & GGTCACACTGGGACAAAG & nd & chr1 & - & 162675015 & none & $1: C>T$ & $3: G>C$ & $5: G>A$ \\
\hline Potential Off-Target site \# 16 & GAACAGACTGGGACAAAG & nd & chr11 & - & 8381632 & none & $2: T>A$ & $3: G>C$ & $5: C>A$ \\
\hline Potential Off-Target site \# 57 & GATGATACTGAGACAAAT & nd & chr16 & - & 87824358 & none & $10: T>C$ & $17: A>C$ & \\
\hline Potential Off-Target site \# 58 & GATGATACTGGGAGAAAA & nd & chr17 & + & \begin{tabular}{|l|}
60562033 \\
\end{tabular} & $\begin{array}{l}\text { TLK2, intronic } \\
\end{array}$ & $13: G>C$ & $17: A>G$ & \\
\hline Potential Off-Target site \# 59 & GATGATACTGGGAGAAAA & nd & chr7 & + & 128177242 & none & $13: G>C$ & $17: A>G$ & \\
\hline Potential Off-Target site \# 91 & GATGATACTGTGATAAAG & nd & chr14 & + & 66373651 & none & $10: T>G$ & $13: T>C$ & \\
\hline Potential Off-Target site \# 92 & GATGATAATGGTACAAAG & nd & chr5 & - & 13605091 & none & $7: T>G$ & $11: A>C$ & \\
\hline Potential Off-Target site \# 93 & GTTGCCACTGGGACAAAG & nd & $\operatorname{chrX}$ & + & 48165879 & RNLP, intronic & $1: T>A$ & $4: C>A$ & $5: C>T$ \\
\hline Potential Off-Target site \# 99 & GACTCTACTGGGACAAAG & nd & chr11 & + & 23900800 & " none & $2: C>T$ & $3: T>G$ & $4: C>A$ \\
\hline $\begin{array}{l}\text { Summary: Off by } 0=1 ; \text { Off by } 1=0 ; \\
\text { Off by } 2=9 ; \text { Off by } 3=143\end{array}$ & & & & & & & & & \\
\hline
\end{tabular}

Total Mutations: $72.71 \%$

Figure S4. On-target and Off-target analysis of the crDMDint2.1: On target activity of the crDMDint2.1 gRNA in myoblasts infected with LV_int2.1 particles. Representative sequences of mutated alleles identified from 110 clonal amplicons. Dashes, deleted bases; red bases, insertions or mutations. Table summary of the on- and off-target analysis based on the ZiFiT software. Only off-targets with two mismatches or three mismatches (red bases) outside the seed gRNA region were tested and are shown. 


\begin{tabular}{|c|c|c|}
\hline Name & Forward & Reverse \\
\hline crDMDex2 & ACACCGGTAAATGCACAATTTTCTAG & AAAACTAGAAAATTGTGCATTTACCG \\
\hline crDMDex2.1 & ACACCGTAAATGCACAATTTTCTAG & AAAACTAGAAAATTGTGCATTTACG \\
\hline crDMDint2.1 & ACACCGATGATACTGGGACAAAGG & AAAACCTTTGTCCCAGTATCATCG \\
\hline crDMDint2.2 & ACACCGTTAAGATTCGGGGACAGG & AAAACCTGTCCCCGAATCTTAACG \\
\hline crDMDint2.3 & ACACCGAGTGGGCTCAGTGTGGGG & AAAACCCCACACTGAGCCCACTCG \\
\hline crDMDint2.4 & ACACCGATAAATGATAATGGAAAG & AAAACTTTCCATTATCATTTATCG \\
\hline crDMDint2.6 & ACACCGACATCAAATCTAGATAAG & AAAACTTATCTAGATTTGATGTCG \\
\hline
\end{tabular}

Table S1: Primers used to clone the DMD gRNAs into MLM3636.

The nucleotides that were removed to clone the gRNAs into the LentiCRISPRv2 are shown in red. 


\begin{tabular}{|c|c|c|}
\hline Name & Sequence & Target \\
\hline $1299 \mathrm{~F}$ & СTGCCTGCTTCTCATAGGACTT & \multirow[t]{2}{*}{ Minimal common region 1} \\
\hline $2562 \mathrm{R}$ & AACACGGAAGGTTTTGCTGATG & \\
\hline $5698 \mathrm{~F}$ & AGTTGTATGAATGTGTGTGTGCC & \multirow[t]{2}{*}{ Minimal common region 2} \\
\hline 6986R & TGGAATTCTGAAGCAGACAAGAGA & \\
\hline $6673 \mathrm{~F}$ & AATTGCTCTGAGGCAGTGCTAA & \multirow[t]{2}{*}{ Minimal common region 3} \\
\hline $7915 \mathrm{R}$ & TGTGCAGGCTTCAATCCATACT & \\
\hline $7555 \mathrm{~F}$ & TAAAGCCTTGGGTTTAAGGGCC & \multirow{2}{*}{ Minimal common region 4} \\
\hline $8710 \mathrm{R}$ & GTGATCCGTCCTCACTGGC & \\
\hline i2.1Offchr5F & CCAGCTGCCGAAGATTCAT & \multirow{2}{*}{ Potential Off-Target site \#92 chr5:13605091 } \\
\hline i2.1Offchr5R & САТСТCCCAGAAACACAGCA & \\
\hline i2.1Offchr14F & ATTCAGGTCCATGGAGGCAG & \multirow{2}{*}{ Potential Off-Target site \#91 chr14:66373651 } \\
\hline i2.1Offchr14R & СтCTTTTCCTTCGCCCTCCC & \\
\hline i2.1Offchr7F & TCTCAGCTCACTGCAACCTC & \multirow{2}{*}{ Potential Off-Target site \#59 chr7:128177242 } \\
\hline $\mathrm{i} 2.1 \mathrm{Offchr} 7 \mathrm{R}$ & TAGCCAGGATGGTCTCGATC & \\
\hline i2.1OffChr17F & AGCAATTCTCGTGCCTCAGC & \multirow{2}{*}{ Potential Off-Target site \#58 chr17:60562033 } \\
\hline i2.1OffChr17R & CACCCACCACCAAGCTCAG & \\
\hline i2.1Offchr16F & AGCTGCTCTTTGGGGTCTTC & \multirow{2}{*}{ Potential Off-Target site \#57 chr16:87824358 } \\
\hline i2.1Offchr16R & GCCTTGTGATCCACTCCCAA & \\
\hline i2.1Offchr2aF & TACCCCAGCAAGGATCCAGA & \multirow{2}{*}{ Potential Off-Target site \#04 chr2:11851832 } \\
\hline i2.1Offchr2aR & CAGAGCAGTGTGAGAGCAGT & \\
\hline i2.1Offchr $12 \mathrm{~F}$ & TGGGTGAGGATGGAGAGGAG & \multirow{2}{*}{ Potential Off-Target site $\# 03 \mathrm{chr} 12: 90417929$} \\
\hline i2.1Offchr12R & ATCCGGGCCCTATGCTATCT & \\
\hline i2.1Offchr $2 \mathrm{bF}$ & TATGGCAGTAGGGGCTGGAT & \multirow{2}{*}{ Potential Off-Target site \#02 chr2:217969247 } \\
\hline i2.1Offchr $2 \mathrm{bR}$ & TCTCTCTGGCTTAGACTTCCA & \\
\hline i2.1Offchr6F & CAGCCTAGGTGACAGAGCAA & \multirow{2}{*}{ Potential Off-Target site \#01 chr6:105368929 } \\
\hline i2.1Offchr6R & GTTGCACTGAGCCGAAGATG & \\
\hline i2.1Offchr1F & GAGACCGTGGTTCCTGTAGC & \multirow{2}{*}{ Potential Off-Target site \#06 chr1:162675015 } \\
\hline i2.1Offchr1R & TAGAGTTGCCCCCTGATCCA & \\
\hline i2.1OffchrXF & CGACCTGCCTCACATACACT & \multirow{2}{*}{ Potential Off-Target site \#93 chrX:48165879 } \\
\hline i2.1OffchrXR & TAACCCGAGATCCAGGCATG & \\
\hline i2.1Offchr $11 \mathrm{aF}$ & AAGGGCCTGACACTTTCCTG & \multirow{2}{*}{ Potential Off-Target site \#16 chr11:8381632 } \\
\hline i2.1Offchr11aR & CCTGAACCCAACAAGACCCT & \\
\hline i2.1Offchr $11 \mathrm{bF}$ & AGCCAATTTTCCTCACACCA & \multirow{2}{*}{ Potential Off-Target site \#99 chr11:23900800 } \\
\hline i2.1Offchr11bR & GTAGCTGGGACTACAGGCAC & \\
\hline
\end{tabular}

Table S2: Primers used for the amplification for the gRNAs on-target and crDMDint2.1 off-target analysis. 


\begin{tabular}{|c|c|c|c|c|}
\hline Name & Sequence & Name & Sequence & Target \\
\hline \multirow{2}{*}{$\underline{1861 \mathrm{~F}}$} & \multirow{2}{*}{ TCTGTTAAAGCAGGCACATTGC } & $2194 \mathrm{~F}$ & GTTTGATGCCAAGAAGGTAGCC & $\mathrm{i} 2.1 / \mathrm{i} 2.2$ inversion \\
\hline & & $2666 \mathrm{R}$ & GTGTCCTGGTTTTCAGTGCATT & i2.1/i2.2 deletion \\
\hline \multirow{2}{*}{$1861 \mathrm{~F}$} & \multirow{2}{*}{ TCTGTTAAAGCAGGCACATTGC } & $5698 \mathrm{~F}$ & AGTTGTATGAATGTGTGTGTGCC & i2.1/i2.3 inversion \\
\hline & & $6154 \mathrm{R}$ & GCCTTTCAGCAGAATTCTAGCC & i2.1/i2.3 deletion \\
\hline \multirow{2}{*}{$1861 \mathrm{~F}$} & \multirow{2}{*}{ TCTGTTAAAGCAGGCACATTGC } & $2194 \mathrm{~F}$ & GTTTGATGCCAAGAAGGTAGCC & i2.1/i2.2 inversion \\
\hline & & $2666 \mathrm{R}$ & GTGTCCTGGTTTTCAGTGCATT & i2.1/i2.2 deletion \\
\hline \multirow[t]{2}{*}{$1861 \mathrm{~F}$} & \multirow{2}{*}{ TCTGTTAAAGCAGGCACATTGC } & $6673 \mathrm{~F}$ & AATTGCTCTGAGGCAGTGCTAA & i2.1/i2.4 inversion \\
\hline & & $6986 \mathrm{R}$ & TGGAATTCTGAAGCAGACAAGAGA & i2.1/i2.4 deletion \\
\hline \multirow{2}{*}{$1861 \mathrm{~F}$} & \multirow{2}{*}{ TCTGTTAAAGCAGGCACATTGC } & $7377 \mathrm{~F}$ & TCTCCTAGTGGATTGTTTTGGCT & i2.1/i2.6 inversion \\
\hline & & $7915 \mathrm{R}$ & TGTGCAGGCTTCAATCCATACT & i2.1/i2.6 deletion \\
\hline \multirow{2}{*}{$1861 \mathrm{~F}$} & \multirow{2}{*}{ TCTGTTAAAGCAGGCACATTGC } & $\underline{8209 \mathrm{~F}}$ & TTTGCCATATCTTCTGCTGCTT & i2.1/ex2-2.1 inversion \\
\hline & & $7915 \mathrm{R}$ & TGTGCAGGCTTCAATCCATACT & i2.1/ex2-2.1 deletion \\
\hline \multirow{2}{*}{$2194 \mathrm{~F}$} & \multirow{2}{*}{ GTTTGATGCCAAGAAGGTAGCC } & $7377 \mathrm{~F}$ & TCTCCTAGTGGATTGTTTTGGCT & i2.2/i2.6 inversion \\
\hline & & $7915 \mathrm{R}$ & TGTGCAGGCTTCAATCCATACT & i2.2/i2.6 deletion \\
\hline \multirow{2}{*}{$5698 \mathrm{~F}$} & \multirow{2}{*}{ AGTTGTATGAATGTGTGTGTGCC } & $7377 \mathrm{~F}$ & TCTCCTAGTGGATTGTTTTGGCT & i2.3/i2.6 inversion \\
\hline & & $7915 \mathrm{R}$ & TGTGCAGGCTTCAATCCATACT & i2.3/i2.6 deletion \\
\hline \multirow{2}{*}{$6673 \mathrm{~F}$} & \multirow{2}{*}{ AATTGCTCTGAGGCAGTGCTAA } & $7377 \mathrm{~F}$ & TCTCCTAGTGGATTGTTTTGGCT & i2.4/i2.6 inversion \\
\hline & & $7915 \mathrm{R}$ & TGTGCAGGCTTCAATCCATACT & i2.4/i2.6 deletion \\
\hline \multirow{2}{*}{$\underline{7377 \mathrm{~F}}$} & \multirow{2}{*}{ TCTCCTAGTGGATTGTTTTGGCT } & $8209 \mathrm{~F}$ & TTTGCCATATCTTCTGCTGCTT & i2.6/ex2-2.1 inversion \\
\hline & & $8710 \mathrm{R}$ & GTGATCCGTCCTCACTGGC & i2.6/ex2-2.1 deletion \\
\hline
\end{tabular}

Table S3: List of primers used to amplify the duplications' and inversions' breakpoints. Primers used to amplify inversions in dup2 myoblasts are underlined in the present table. 